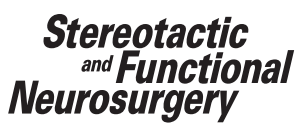

Stereotactic

Neurosurgery
Stereotact Funct Neurosurg 2010;88:281-287

DOI: $10.1159 / 000316760$
Received: April 13, 2009

Accepted after revision: January 11, 2010

Published online: June 24, 2010

\title{
Closed-Loop Stimulation in the Control of Focal Epilepsy of Insular Origin
}

\author{
Joseph R. Smith ${ }^{a}$ Kostas N. Fountas ${ }^{d}$ Anthony M. Murro ${ }^{b}$ Yong D. Park $^{b}$ \\ Patrick D. Jenkins ${ }^{a}$ Martha Morrell ${ }^{c}$ Rosanna Esteller ${ }^{c}$ David Greene ${ }^{c}$ \\ ${ }^{a}$ Department of Neurosurgery, and b ${ }^{b}$ Department of Neurology, Medical College of Georgia, Augusta, Ga., and \\ ${ }^{c}$ Neuropace, Inc., Mountain View, Calif., USA; ${ }^{d}$ Department of Neurosurgery, University of Thessaly School of \\ Medicine, Terpsithea-Larissa, Greece
}

\section{Key Words}

Insular epilepsy · Depth electrode localization • Responsive neurostimulator

\begin{abstract}
Background: Previous studies have shown that closed-loop or responsive neurostimulation can abort induced or spontaneous epileptiform discharges. Objective: To assess the effectiveness of a programmable cranially implanted closedloop neurostimulation system in the control of seizures originating from an area relatively inaccessible by open craniotomy. Method: A patient with drug-resistant partial epilepsy had previously undergone open resection of the left frontal opercular cortex and the underlying insular area. Although subdural-depth electrode ictal recordings had been nonlocalizing, depth electrode insular stimulation had produced the patient's habitual aura. Postoperatively, there was a sustained $50 \%$ reduction in seizure frequency. The residual seizures were identical to the preoperative seizures. Repeat depth electrode monitoring revealed that the ictal focus was immediately posterior to the previously resected insular area. A closed-loop cranial internal pulse generator system including left anterior insular and posterior orbitofrontal depth electrodes was implanted. Result: There was an ad-
\end{abstract}

ditional $60 \%$ reduction of seizures. Conclusion: Preliminary observation indicates that responsive neurostimulation may be an effective alternative to higher-risk resective epilepsy surgery.

Copyright $\odot 2010$ S. Karger AG, Basel

\section{Introduction}

Uncontrolled open-loop stimulation studies in adults with medically intractable epilepsy have shown varying control of seizures with stimulation of the cerebellar cortex [1], cerebellar dentate nucleus [2], anterior thalamic nucleus [3], centromedian thalamic nucleus [4], caudate head [2], hippocampus [2, 5], and subthalamic nucleus [6]. The only controlled studies involved the cerebellar cortex [7] and thalamic centromedian nucleus stimulation [8], and neither showed that stimulation had a significant effect on clinical seizures. However, the vagus nerve stimulator, which provides scheduled cyclical open-loop stimulation to the left vagus nerve, has been shown to reduce seizures in several controlled clinical trials [9].

One study showed that stimulation-induced cortical afterdischarges could be terminated by applying respon-

\section{KARGER}

Fax +4161306 1234 E-Mail karger@karger.ch www.karger.com

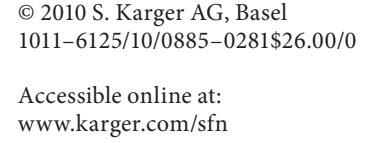

Tel. +1 706721 3071, Fax +1 706721 8084,E-Mail jsmith@ mail.mcg.edu 
sive stimulation [10]. The authors entertained the possibility that an implanted closed-loop device could both detect and terminate epileptiform activity. Subsequent clinical trials assessed patient response to closed-loop systems that provided stimulation contingent on seizure detection [11-13]. One multicenter study utilized an external responsive device that could be programmed to detect and deliver therapeutic stimulation to areas generating epileptiform activity. This study provided preliminary information that responsive stimulation was well tolerated and that some epileptiform discharges could be altered [12]. Based on these results, clinical trials with an implantable device that provided responsive neurostimulation (the RNS ${ }^{\mathrm{TM}}$ System) were begun.

Subjects included in the RNS System clinical investigations have been adults with medically intractable disabling partial-onset seizures localized to 1 or 2 epileptic foci. Safety and efficacy were examined in a recently completed pivotal clinical investigation [unpubl. data]. Experience with 1 subject participating in a previously completed multicenter feasibility clinical investigation [27] is provided here and suggests that the RNS System might be a useful treatment approach for patients whose seizure focus is within an area relatively difficult to access with open craniotomy.

Utilizing stereotactic depth electrodes, we identified a patient with residual postoperative seizures originating in the left anterior superior insular area. Because of the risk to language function when approaching the anterior insula in the language-dominant hemisphere $[14,15]$, and because stereotactic depth electrodes could be safely implanted in the insular area [16], we presented the surgical option of implanting a closed-loop stimulation device to this patient.

\section{Materials and Methods}

Candidacy, surgical technique, and the RNS System have been previously described [17]. As with other candidates for RNS System implantation, the current patient had focal seizures that had not responded adequately to multiple antiepileptic medications over an extended period. Also, as with other candidates, the present patient had had at least 4 disabling seizures per month over a 3-month period before final consideration for implantation.

The neurostimulator system consists of 3 components: (1) an internal pulse generator which contains electronics, a telemetry coil, a battery, and a connector port that accommodates one or two 4-contact electrodes; (2) quadripolar stereotactic depth electrodes with $1.27-\mathrm{mm}$ diameter by $1.5-\mathrm{mm}$-long platinum-iridium contacts spaced 3.5 or $10 \mathrm{~mm}$ apart, and total lengths of 30 or 44 $\mathrm{cm}$, and (3) a handheld computer with specialized programming software and a telemetry wand that communicates with the internal pulse generator.

The RNS system continuously analyzes electrocorticography and triggers electrical stimulation when it detects specific characteristics programmed by the clinician as indicative of epileptiform activity. It also stores and can download diagnostic information that details detections and stimulations, including multichannel stored electrocorticography. The neurostimulator system has a limited electrocorticography memory buffer. The number of electrocorticographies stored depends on the number of recording channels and the recording time selected. Typically, 2 bipolar recording channels are selected with a 60 -second pretrigger and 30-second posttrigger duration, which allows 9 electrocorticographies to be stored. Any additional electrocorticographies overwrite the previous recordings. Electrocorticography storage can be triggered by any of several electrographic events, including seizure onset.

The neurostimulator system utilizes any of 3 seizure detection tools (line length, area, and half-wave tools) operating on 1 or 2 detection channels. The line length and area tools compare the electrographic activity contained within a short-term sample time window to that contained within a longer-term trend window. When activity within the sample window exceeds the trend activity by a specified percentage, detection occurs. The line length tool is more commonly used to detect high-frequency, lowamplitude activity that diverges in amplitude little from the baseline electrographic amplitude, but has significant summed line length [18]. The area tool is more commonly used for slower rhythmic, higher amplitude epileptiform activity with large integrated areas. The half-wave tool measures half-waves of specified duration, amplitude, and frequency bandwidth [19]. When an epileptiform discharge meeting these criteria is detected, programmed therapeutic stimulation is delivered.

Therapeutic stimulation consists of charge-balanced biphasic pulses with amplitude programmable from 0.5 to $12 \mathrm{~mA}$, pulse width programmable from 40 to $1,000 \mu \mathrm{s}$, frequency programmable from 1 to $333 \mathrm{~Hz}$, and pulse trains or bursts programmable from 10 to 5,000 ms. Any of the electrode contacts or the pulse generator housing may be programmed as anode or cathode. After a pulse train therapy has been delivered, a redetection algorithm determines if the epileptiform activity is still present. If so, up to 4 additional therapies may be delivered per episode. Also, each therapy may consist of 1 or 2 pulse trains. The parameters of each therapy and each pulse train may be the same or varied. The neurostimulator system has a built-in charge density limit that will allow no more than $25 \mu \mathrm{C} / \mathrm{cm}^{2} /$ phase charge density to be delivered.

\section{Case Report}

The patient was a 33-year-old male whose seizures began at the age of 12 years. These were characterized by an aura of epigastric tightening followed by extension at the right elbow, grasping with the left hand, then kicking with 1 or both legs and vocalization. The seizures lasted 5-10 s during which the patient remained conscious with intact speech comprehension. Multiple antiepileptic medications had failed to significantly affect seizure frequency and intensity. 


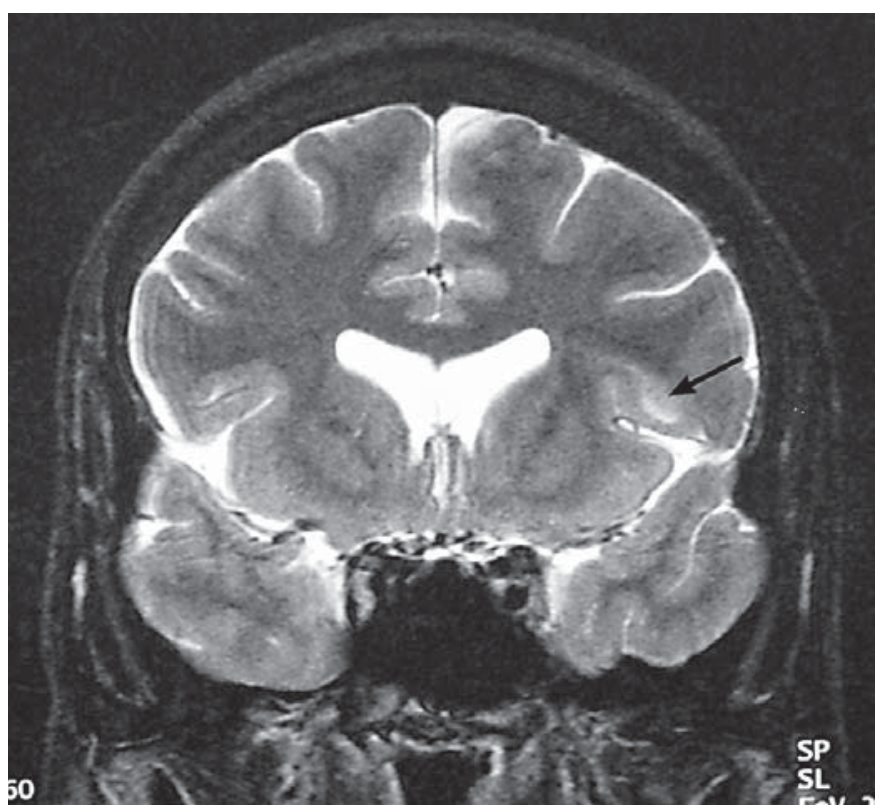

Fig. 1. MRI shows the area of increased signal in the left frontal operculum (arrow).

Neurological examination was normal. Neuropsychological testing revealed a full-scale IQ score of 104, a verbal IQ score of 103 and a visuospatial IQ score of 106. Delayed verbal memory score was 114 , and visuospatial memory score was 103 . Intracarotid sodium amytal (Wada) testing revealed left language dominance with object recall of 4 of 8 items after left injection and 8 of 8 items after right injection.

Video EEG monitoring showed interictal discharges at F3 and F4. Multiple ictal EEG recordings did not localize the seizure focus. Brain MRI showed increased $\mathrm{T}_{2}$ signal in the subcortical white matter of the left frontal opercular area (fig. 1). SISCOM (subtraction ictal SPECT coregistered to MRI) showed increased isotope uptake in the left mesial frontal, left insulo-opercular, and right insular areas.

Subsequent continuous video EEG monitoring utilized stereotactic depth electrodes in left mesial frontal and insular areas, as well as subdural electrodes including a $6 \times 8$ left frontal convexity grid, and anterior and posterior left orbitofrontal and mesial frontal $1 \times 4$ strip electrodes (fig. 2). The insular electrode was included because the patient described an aura of epigastric tightening [20], and because of hypermotor activity (kicking) [21, 22]. There was no localized interictal activity at any of the electrode contacts and ictal recordings revealed diffuse nonlocalized attenuation. Subsequent extraoperative stimulation studies showed that stimulation at contact 2 of the left insular electrode (fig. 2) produced the patient's habitual epigastric aura. Stimulation at contact 3 produced a cephalic sensation and stimulation at contact 1 produced no phenomena. Intraoperative stimulation map-

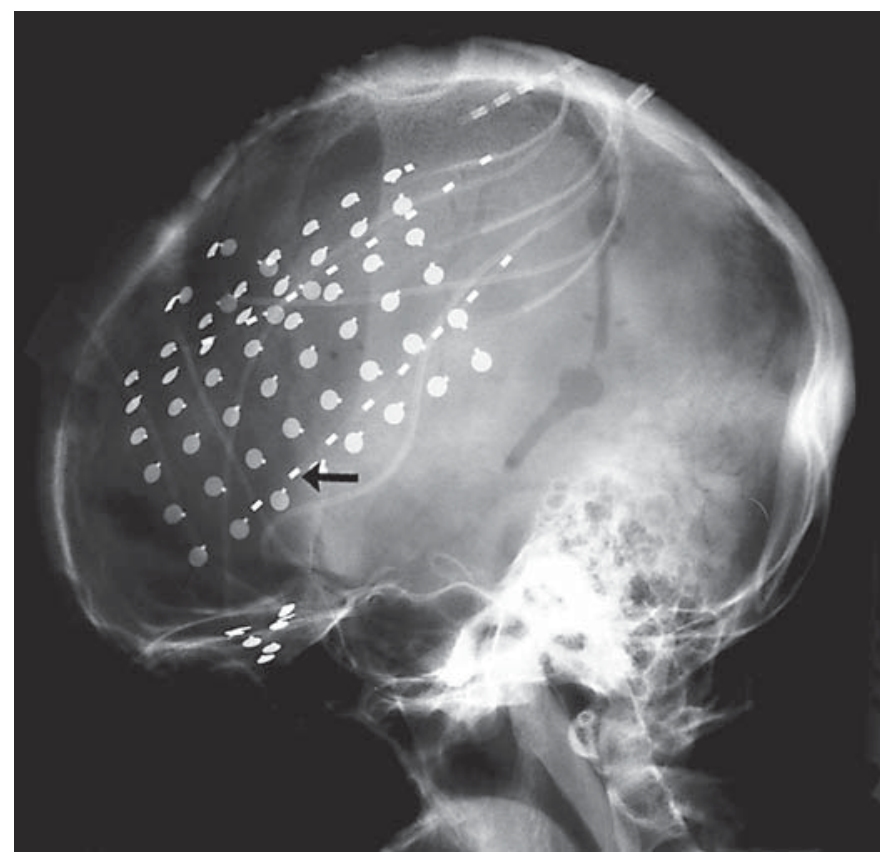

Fig. 2. Arrow points to contact 2 of the insular depth electrode. In addition, a $6 \times 8$ left frontal convexity subdural electrode, a $1 \times$ 8 left mesial frontal depth electrode, and two $1 \times 4$ mesial frontal and two orbitofrontal $1 \times 4$ subdural strip electrodes were implanted.

ping at the time of electrode implantation had produced no naming errors, although left language dominance was noted on previous Wada testing.

The patient returned to surgery. After removal of the electrodes, the left anterior frontal opercular cortex was resected to access the subjacent insula where stimulation had reproduced the patient's epigastric aura. This insular area was then resected (see sagittal reconstruction in fig. 3). Although language mapping of the more posterior frontal operculum (the area of subcortical increased $\mathrm{T}_{2}$ signal) had not produced any language errors, this area was spared as recommended by Rasmussen [23]. Histopathologic examination of the opercular specimen revealed only gliosis. Histologic examination of the anterior insular tissue showed cortical dysplasia.

When the patient was re-evaluated 3 years later, his habitual seizure frequency had decreased by $50 \%$. No epileptiform activity was discernible on interictal or ictal EEG. Subsequent magnetoencephalography showed multiple interictal epileptiform dipoles in the left anterior insular area (fig. 4). Stereotactic left orbitofrontal and anterior insular depth electrodes were placed to assess the possibility of additional epilepsy surgery. Multiple electrographic (and clinical) seizure onsets were recorded from contacts 4 and 5 of the insular electrode with rapid spread to contacts 6 and 7 of the orbitofrontal electrode (fig. 5). Further resection of the anterior insula through a transopercular approach was thought too risky to language function, so the patient was subsequently enrolled in the RNS System feasibility investigation. 
Fig. 3. Post-depth electrode implant $\mathrm{T}_{2^{-}}$ weighted MRI (later fused to stereotactic CT and $\mathrm{T}_{1}$-weighted MRI obtained at the time of RNS implant). The depth electrode contacts are immediately posterior to the trajectories constructed for the RNS depth electrodes. The insular resection (arrow) is between the two trajectories on the sagittal reconstruction.
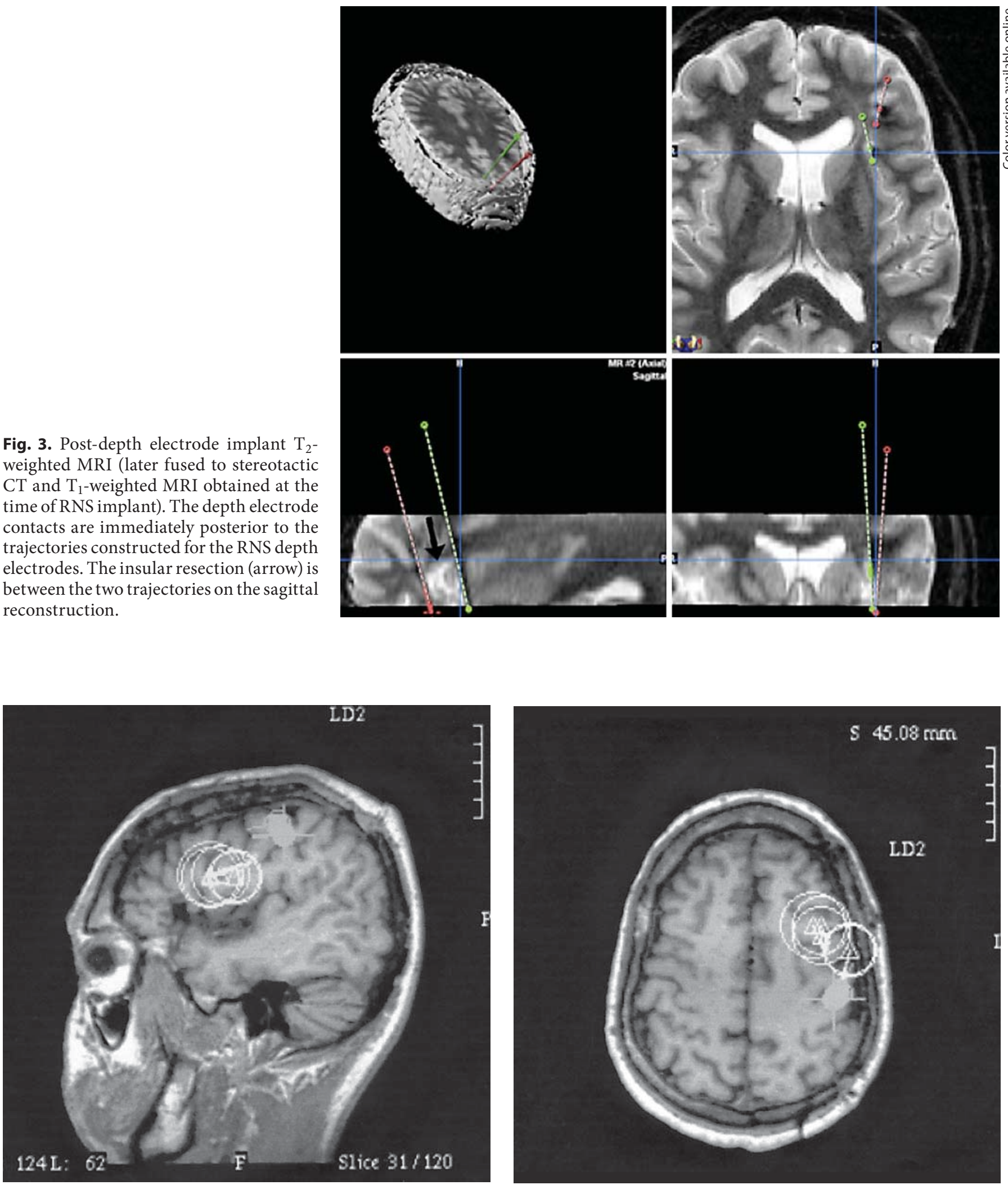

Fig. 4. Encircled white triangles represent interictal epileptiform magnetic dipoles located in the region of the left anterior superior insula. 


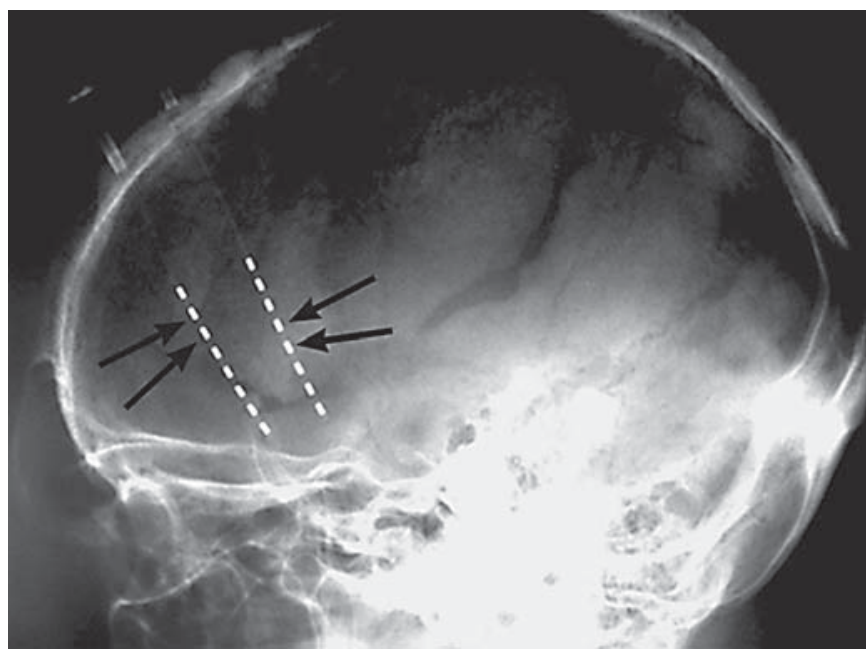

Fig. 5. Anterior arrows point to contacts 6 and 7 of the left orbitofrontal depth electrode. Posterior arrows point to contacts 4 and 5 of the insular depth electrode.

Fig. 7. Example of aborted epileptiform activity recorded from channels 1 and 2 (recorded from insular depth electrode contacts 1-2 and 3-4, respectively). Label B indicates point of seizure detection. Beginning of therapeutic stimulation delivery is labeled Tr. Subsequent electrocorticography recording shows reversion to nonepileptiform pattern.

An MRI was obtained (fig. 3) prior to removal of the depth electrodes to document the exact site of the contacts involved in seizure localization. This study was later fused with stereotactic $\mathrm{CT}$ and contrast-enhanced magnetization-prepared rapid gradient echo MRI scans at the time the RNS System neurostimulator and two 4-contact stereotactic depth electrodes (10-mm contact spacing) were implanted (fig. 6).

The RNS System neurostimulator utilized 2 seizure detection tools: the line length [18] and half-wave tools [17]. The line length tool employed a detection threshold of $112 \%$ of baseline line length. The half-wave tool employed an amplitude threshold of $5 \%$ of peak spike amplitude, half-wave duration threshold of $5 \%$ of maximum, and a frequency range of 15-42 Hz. Cathodal stimulation was de-

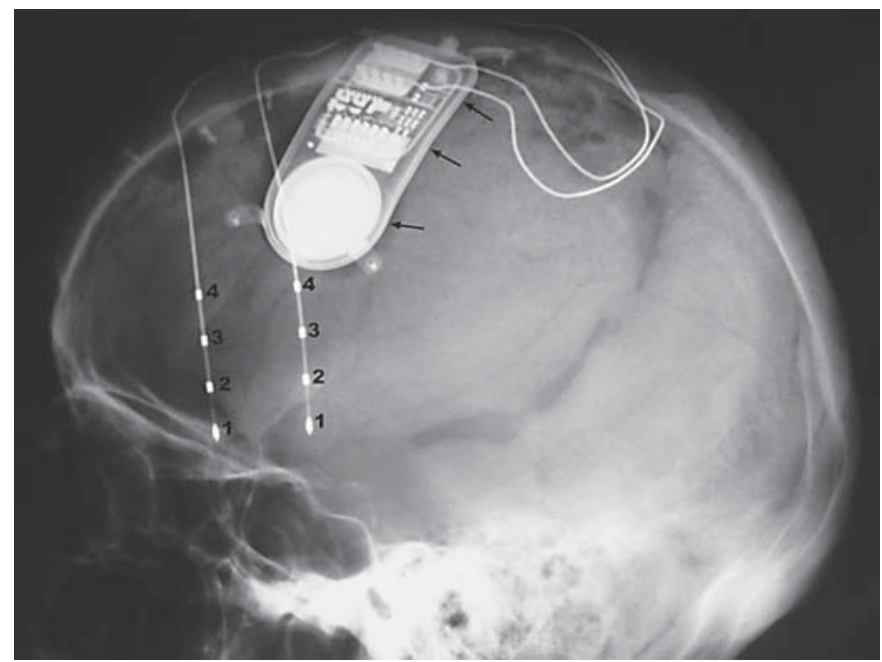

Fig. 6. The anterior orbitofrontal and more posterior insular depth electrode contacts are numbered. The upper arrow points to the connector port of the RNS system, the middle arrow to the microprocessor, and the lower arrow to the battery.

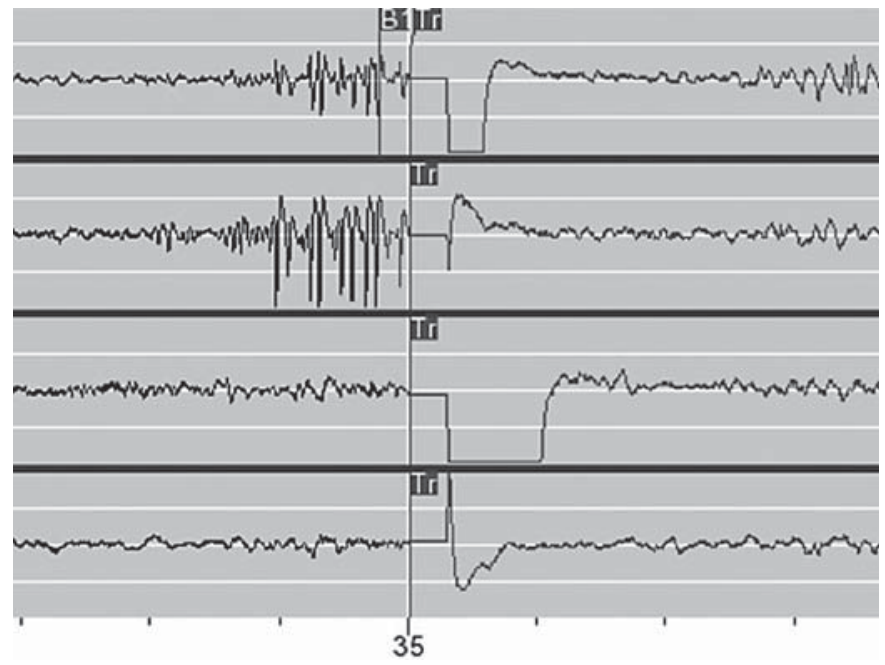

livered through contacts 1-4 of the insular electrode. Stimulation parameters were as follows: current $=8 \mathrm{~mA}$, frequency $=20 \mathrm{~Hz}$, pulse width $=120 \mu \mathrm{s} /$ phase and pulse train duration $=50 \mathrm{~ms}$. Figure 7 shows an example of an epileptiform discharge that received stimulation and did not develop into an electrographic or clinical seizure. Since implantation of the RNS System neurostimulator and leads, the seizure frequency has been reduced by an additional 60\% (median 18 seizures/month before implantation, median 7 seizures/month over the most recent 2 years) for a total of $80 \%$ seizure reduction since the initial resective surgery. Figure 8 shows in graphic format the decrease in seizure frequency over the nearly 4 -year postoperative period. 
Fig. 8. The bar graph shows the seizure frequency both before (preop) and after implantation of the RNS System.

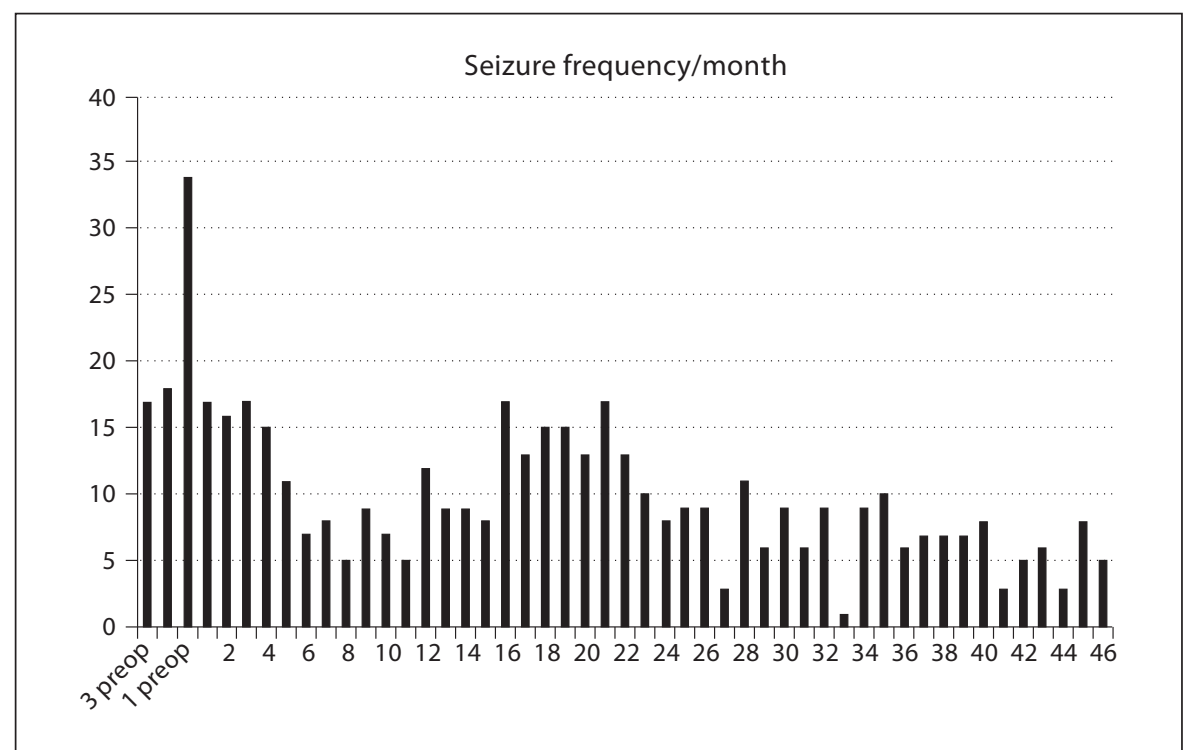

\section{Conclusions}

Studies on the effect of electrical stimulation on induced cortical afterdischarges have shown that afterdischarges can be terminated sometimes [10], and that there may be optimal parameters for this [24]. External responsive neurostimulation studies have revealed that closedloop stimulation can alter some spontaneously occurring electrographic seizure activity $[11,12,25]$. Early seizure detection and stimulation of multiple contacts in the immediate vicinity of the seizure focus appears to increase the likelihood that the epileptiform activity can be suppressed [24]. An implantable device, the RNS System, has been tested in multicenter controlled clinical trials as an adjunctive therapy for adults with medically intractable disabling partial seizures in order to assess safety and to determine efficacy [26]. The present report suggests that this closed-loop system may be effective in controlling medically intractable focal epilepsy that originates in areas that are relatively difficult to access with resective surgery.

Intractable focal epilepsy of insular origin may be more common than originally thought. It has been suggested that as many as $10 \%$ of seizures thought to be of mesial temporal origin may originate from the insula [20]. Since resection of such foci has inherent dangers, some have suggested stereotactic radiofrequency lesioning as an alternative [20]. Based on our initial experience with the currently reported patient, closed-loop cortical stimulation may prove to be a reasonable alternative.

\section{References}

1 Cooper IS, Upton AR: Effects of cerebellar stimulation on epilepsy, the EEG and cerebral palsy in man. Electroencephalogr Clin Neurophysiol 1978;34:349-354.

-2 Sramka M, Fritz G, Galanda M, Nadvornik P: Some observations in treatment stimulation of epilepsy. Acta Neurochir (Wien) 1976;23(suppl):257-262.

- 3 Kerrigan JF, Litt B, Fisher RS, Cranstoun S, French JA, Blum DE, Dichter M, Shetter A, Baltuch G, Jaggi J, Krone S, Brodie M, Rise M, Graves N: Electrical stimulation of the anterior nucleus of the thalamus for the treatment of intractable epilepsy. Epilepsia 2004;45:346-354.
4 Velasco F, Velasco M, Jimenez F, Velasco AL, Marquez I: Stimulation of the central median thalamic nucleus for epilepsy. Stereotact Funct Neurosurg 2001;77:228-232.

5 Velasco F, Velasco M, Velasco AL, Menez D, Rocha L: Electrical stimulation for epilepsy: stimulation of hippocampal foci. Stereotact Funct Neurosurg 2001;77:223-227.

6 Benabid AL, Koudsie A, Chabardes S, Vercueil L, Benazzouz A, Minotti L, Le Bas JF, Kahane P, de Sanit Martin A, Hirsch E: Subthalamic nucleus and substantia nigra pars reticulata stimulation: the Grenoble experience; in Luders HO (ed): Deep Brain Stimulation and Epilepsy. London, Dunitz, 2004, pp 335-348. 
7 Van Buren JM, Wood JH, Oakley J, Hambrecht F: Preliminary evaluation of cerebellar stimulation by double-blind stimulation and biological criteria in the treatment of epilepsy. J Neurosurg 1978;48:407-416.

$>8$ Fisher RS, Uematsu S, Krauss GL, Cysyk BJ, McPherson R, Lesser RP, Gordon B, Schwerdt P, Rise M: Placebo-controlled pilot study of centromedian thalamic stimulation in treatment of intractable seizures. Epilepsia 1992;33:841-851.

9 Labar D: Vagal nerve stimulation: effects on seizures; in Luders HO (ed): Deep Brain Stimulation and Epilepsy. London, Dunitz, 2004, pp 255-262.

-10 Lesser RP, Kim SH, Beyderman L, Miglioret ti DL, Webber WR, Bare M, Cysyk B, Krauss $\mathrm{G}$, Gordon B: Brief bursts of pulse stimulation terminate afterdischarges caused by cortical stimulation. Neurology 1999;53: 2073-2081.

11 Murro A, Park Y, Greene D, Smith J, Ray P, King D, Loring D, Lee K: Closed loop neurostimulation in patient with intractable epilepsy. Annu Meet Am Clin Neurophysiol Soc, New Orleans, 2002.

12 Murro AM, Park YD, Bergey GK, Kossoff EH, Ritzl EK, Karceski SC, Flynn K, Choi H, Spencer DD, Duckrow RB, Seale C: Multicenter study of acute responsive stimulation in patients with intractable epilepsy. Epilepsia 2003;44:326.

13 Osorio I, Frei MG, Manly BF, Sunderam S, Bhavaraju NC, Wilkinson SB: An introduction to contingent (closed-loop) brain electrical stimulation for seizure blockage, to ultra-short-term clinical trials, and to multidimensional statistical analysis of therapeutic efficacy. J Clin Neurophysiol 2001;18: 533-544.
14 Dobesberger J, Ortler M, Unterberger I, Walser G, Falkenstetter T, Bodner T, Benke T, Bale R, Fiegele T, Donnemiller E, Gotwald T, Trinka E: Successful surgical treatment of insular epilepsy with nocturnal hypermotor seizures. Epilepsia 2008;49:159-162.

15 Duffau H, Capelle L, Lopes M, Faillot T, Sichez JP, Fohanno D: The insular lobe: physiopathological and surgical considerations. Neurosurgery 2000;47:801-810.

16 Afif A, Chabardes S, Minotti L, Kahane P, Hoffmann D: Safety and usefulness of insular depth electrodes implanted via an oblique approach in patients with epilepsy. Neurosurgery 2008;62:479-480.

17 Fountas KN, Smith JR, Murro AM, Politsky J, Park YD, Jenkins PD: Implantation of a closed-loop stimulation in the management of medically refractory focal epilepsy: a technical note. Stereotact Funct Neurosurg 2005; 83:153-158.

18 Esteller R, Echauz J, Tcheng T, Litt B, Pless B: Line length: an efficient feature for seizure onset detection. Proc 23rd Annu Int Conf IEEE Eng Med Biol Soc, Istanbul, 2001, pp 1707-1710.

19 Gotman J: Automatic recognition of epileptic seizures in the EEG. Electroencephalogr Clin Neurophysiol 1982;54:530-540.

20 Guenot M, Isnard J: Epilepsy and insula. Neurochirurgie 2008;54:374-381.
21 Kaido T, Otsuki T, Nakama H, Kaneko Y, Kubota Y, Sugai K, Saito O: Complex behavioral automatism arising from insular cortex. Epilepsy Behav 2006;8:315-319.

-22 Ryvlin P, Minotti L, Demarquay G, Hirsch E, Arzimanoglou A, Hoffman D, Guenot M, Picard F, Rheims S, Kahane P: Nocturnal hypermotor seizures, suggesting frontal lobe epilepsy, can originate in the insula. Epilepsia 2006;47:755-765.

23 Rasmussen T: Surgery of frontal lobe epilepsy; in Purpura DP, Penry JK, Walter RD (eds): Advances in Neurology: Neurosurgical Management of the Epilepsies. New York, Raven Press, 1975, vol 8, pp 197-205.

24 Motamedi GK, Lesser RP, Miglioretti DL, Mizuno-Matsumoto Y, Gordon B, Webber WR, Jackson DC, Sepkuty JP, Crone NE: Optimizing parameters for terminating cortical afterdischarges with pulse stimulation. Epilepsia 2002;43:836-846.

25 Peters TE, Bhavaraju NC, Frei MG, Osorio I: Network system for automated seizure detection and contingent delivery of therapy. J Clin Neurophysiol 2001;18:545-549.

26 Smith JR, Fountas KN, Murro AM, Park YD, Jenkins PD, Greene DRE: Closed-loop stimulation in the control of focal epilepsy; in Krames E, Peckham PH, Rezai AR (eds): Textbook of Neuromodulation. Amsterdam, Elsevier, 2009, vol 2, pp 657-662.

27 Morrell M, Hirsch L, Bergey G, Barkley G, Wharen R, Murro AM, Fisch B, Rossi MA, Labar D, Duckrow R, Sirvin JI, Drazkowski J, Worrell GA, Gwinn RP: Long-term safety and efficacy of the rns(tm) system in adults with medically intractable partial onset seizures. Epilepsia 2008;49(suppl 7):480-481. 